SABRAO Journal of Breeding and Genetics

53 (4) 749-757, 2021

http://doi.org/10.54910/sabrao2021.53.4.16

http://sabraojournal.org/

pISSN 1029-7073; eISSN 2224-8978

\title{
VALIDATION OF MOLECULAR MARKERS LINKED TO CERCOSPORA LEAF SPOT DISEASE RESISTANCE IN MUNGBEAN (Vigna radiata [L.] WILCZEK)
}

\author{
P. PAPAN ${ }^{1}$, W. CHUEAKHUNTHOD ${ }^{1}$, O. POOLSAWAT ${ }^{1}$, K. ARSAKIT $^{1}$, \\ A. THARAPREUKSAPONG ${ }^{2}$ and P.A. TANTASAWAT ${ }^{1 *}$
${ }^{1}$ School of Crop Production Technology, Institute of Agricultural Technology, Suranaree University of Technology, Nakhon Ratchasima, Thailand
${ }^{2}$ Center for Scientific and Technological Equipment, Suranaree University of Technology, Nakhon Ratchasima, Thailand
*Corresponding author email: piyada@sut.ac.th
Email addresses of co-authors: pichakon.111@gmail.com, witsarut.c@sut.ac.th, oythip@gmail.com, kitiya230@gmail.com, akkawat@sut.ac.th

\section{SUMMARY}

Cercospora leaf spot (CLS) resistance is a highly desirable trait for mungbean (Vigna radiata [L.] Wilczek) production in Thailand. 'V4718' is a vital resistance source that shows high and stable resistance to CLS disease. A previous study identified a major quantitative trait locus (QTL) ( $q C L S C 72$ V18-1) controlling CLS resistance and found the marker (I16274) that was located closest to the resistance gene by using $F_{2: 9}$ and $F_{2: 10}$ recombinant inbred line populations derived through a cross between 'V4718' and the susceptible variety 'Chai Nat 72' ('CN72'). Here, we evaluated three newly reported simple sequence repeat (SSR) markers and one InDel marker together with six previously identified markers that were linked to $q C L S C 72 V 18-1$ to further identify the markers that were located close to this QTL. By performing bulk segregant analysis on two validation populations, we found that two SSR markers (Vr6gCLS037 and Vr6gCLS133) and one InDel marker (VrTAF5_indel) were putatively associated with CLS resistance. Of these markers, only the VrTAF5_indel marker showed a significant association with the CLS resistance gene with a logarithm of odds score $>3$ across the phenotypic data for 2016 and 2018. QTL analysis with inclusive composite interval mapping revealed that the VrTAF5_indel marker was integrated into the genetic map with other previously identified markers. The I16274 and VrTAF5_indel markers flanking the QTL of interest accounted for $41.56 \%-60.38 \%$ of the phenotypic variation with genetic distances of 4.0 and $5.0 \mathrm{cM}$ from the resistance gene, respectively. Both markers together permitted only $0.40 \%$ recombination with the CLS resistance gene in markerassisted selection and thus could be useful in future breeding efforts for CLS resistance in mungbean.

Keywords: Cercospora leaf spot, inclusive composite interval mapping, marker-assisted selection, mungbean, quantitative trait loci analysis

Key findings: This study identified that the I16274 and VrTAF5_indel markers flanked qCLSC72V18-1 and conferred CLS resistance to 'V4718'. Both markers, which together allowed rapid selection and only $0.40 \%$ recombination with the CLS resistance gene in marker-assisted selection, are useful for developing CLS-resistant mungbean varieties. 
Manuscript received: August 17, 2021; Accepted: September 25, 2021.

(C) Society for the Advancement of Breeding Research in Asia and Oceania (SABRAO) 2021

Communicating Editor: Prof. P.I. Prasanthi Perera

\section{INTRODUCTION}

Mungbean (Vigna radiata [L.] Wilczek) is an economically important legume crop in Asian countries, mainly India, China, Myanmar, Indonesia, Bangladesh, Pakistan, and Thailand, which cultivate over $90 \%$ of mungbean worldwide (Ruanpanun and Somta, 2021). This crop is utilized in several ways. For example, mungbean seeds, which contain starch, digestible proteins, minerals, vitamins, and amino acids, are used in industrial foods (vermicelli and starch) and cosmetics, while sprouts and young pods are eaten as vegetables. In addition, mungbean leaves and stems are applied as forage and green manure. However, mungbean production is dramatically constrained by various factors, i.e., susceptibility to pests and diseases or environmental weaknesses.

Cercospora leaf spot (CLS), a severe foliar disease capable of inducing leaf spot and defoliation, is caused by Cercospora canescens Ellis \& Martin (Chand et al., 2015). This disease spreads to mungbean fields particularly during the warm-wet growing season and often leads to $50 \%$ economic losses in the absence of protection (AVRDC, 1984). The deployment of resistant varieties is the most efficient and durable strategy for the integrated management of this disease. By screening CLS-resistant mungbean genotypes from several countries, Nair et al. (2019) identified several genotypes with resistance to CLS: 'NM-1', 'NM-2', 'NM-98', 'BRM-188', 'BARIMung-2', 'C2/94-4-42', '98-cmg-003', '98-cmg018 ', 'Basanti', 'PDM-11', 'CO-3', and 'VC3960-88' (Iqbal et al., 2004). Other genotypes with CLS resistance include 'M5-22' and 'M5-25' (Wongpiyasatid et al., 1999), as well as 'V1471', 'V2773', 'V2757', 'V5036', and 'V4718' (Hartman et al., 1993). However, only a few resources, including 'V4718' (Hartman et al., 1993;
Chankaew et al., 2011; Arsakit et al., 2017; Chueakhunthod et al., 2020; Tantasawat et al., 2020; Yundaeng et al., 2020), have been identified to provide high and stable resistance to CLS. The genetic inheritance of CLS resistance has been reported elsewhere and is either controlled by qualitative (Mishra et al., 1988; Chankaew et al., 2011; Tantasawat et al., 2020) or quantitative genes (AVRDC, 1980; Leabwon and Oupadissakoon, 1984) in accordance with the resistance source. A previous study discovered a major quantitative trait locus (QTL) associated with CLS resistance by using simple sequence repeat (SSR) markers in a cross generated by hybridizing 'CN72' (a susceptible variety) and 'V4718'. This QTL is located between the VR393 and CEDG084 markers (Arsakit et al., 2017). Later, Tantasawat et al. (2020) identified the inter-simple sequence repeat (ISSR) marker I16274, which is closer than CEDG084 to the resistance gene that increases the efficiency of selection for the resistance gene through marker-assisted selection (MAS) with VR393 and I16274. By using a SSR marker system, Chankaew et al. (2011) also found a major QTL that controls resistance to CLS in another cross between 'Kamphaeng Saen 1' ('KPS1'), a susceptible variety, and 'V4718'. This QTL is flanked by the CEDG117 and VR393 markers.

The evolution of genomic sequencing or transcriptomic sequencing throughout the genome has influenced the trend of the use of structural markers in lieu of functional markers (Poczai et al., 2013). Functional markers located at or near any genes are very useful for selection. By using this technique on a 'KPS1' $\times$ 'V4718' cross, Yundaeng et al. (2020) identified TATA-binding-proteinassociated factor 5 , a subunit of the transcription initiation factor IID and SptAda-Gcn5 acetyltransferase complexes 
that are encoded by a candidate gene responsible for CLS resistance (VrTAF5). Moreover, they found two InDel (VrTAF5_indel) and SSR (Vr6gCLS085) markers that flank the functional gene VrTAF5. These VrTAF5_indel and Vr6gCLS085 markers are only 12 and 13 $\mathrm{Kb}$ from VrTAF5 and are thus closer than the previously identified flanking markers CEDG117 and VR393. The markers associated with CLS resistance can be used to accelerate the development of new resistant varieties through year-round MAS. In this study, we identified the closest markers and refined QTL mapping for resistance to CLS in a recombinant inbred line (RIL) population of mungbean obtained from a cross between 'CN72' and 'V4718'.

\section{MATERIALS AND METHODS}

\section{Mapping population and CLS disease data}

The population of $143 \mathrm{~F}_{2: 9}$ and $\mathrm{F}_{2: 10}$ RILs of the 'CN72' $\times$ 'V4718' cross was obtained from Khajudparn (2009), who developed the population via the single-seed descent method. 'CN72' is a cultivated mungbean variety with a high yield in Thailand but high susceptibility to CLS, whereas 'V4718' is a resistant Indian mungbean line obtained from the World Vegetable Center in Taiwan.

The disease scores for the 2016 and 2018 data of the $F_{2: 9}$ and $F_{2: 10}$ RIL populations from Tantasawat et al. (2020) were used for QTL analysis. Briefly, both populations and their parents were planted in a randomized complete block design with three replicates during the rainy season (May to August) in 2016 and 2018 under field conditions at the Suranaree University of Technology, Nakhon Ratchasima, Thailand. In each block, the seeds of each RIL were planted in a single $2 \mathrm{~m}$-long row with an intra-row spacing of $20 \mathrm{~cm}$ and inter-row spacing of $50 \mathrm{~cm}$. Two plants per hill (ca. 20 plants per row) were retained. The susceptible parent 'CN72' variety was additionally grown around the blocks as a source of CLS inoculum. The severity of CLS in all RILs and parental lines was observed at 65 days after planting by using the scoring system described by Chankaew et al. (2011).

\section{DNA marker analysis}

Three SSR primer pairs (Vr6gCLS037, Vr6gCLS085, and Vr6gCLS133) and one InDel primer pair (VrTAF5_indel) flanking qCLS on chromosome 6 of the 'KPS1' $\times$ 'V4718' cross from Yundaeng et al. (2020) were used for initial screening with bulk segregant analysis (BSA). This technique was carried out by using the DNA of 'CN72' (S), 'V4718' (R), the resistant bulk (RB), and the susceptible bulk (SB). Polymerase chain reaction (PCR) for SSR and InDel analysis was performed by using a $20 \mu \mathrm{l}$ reaction mixture containing $2 \mathrm{ng}$ of the genomic DNA template, $1 \times$ buffer $(50 \mathrm{mM} \mathrm{KCl}, 10 \mathrm{mM}$ Tris- $\mathrm{HCl}, \mathrm{pH}$ 9.1, $0.01 \%$ Triton $\left.^{\mathrm{TM}} \mathrm{X}-100\right), 2 \mathrm{mM} \mathrm{MgCl}$, $0.2 \mathrm{mM}$ each dNTP, and 1 unit of Taq DNA polymerase (Vivantis, Selangor Darul Ehsan, Malaysia), and $0.5 \mu \mathrm{M}$ each of the forward and reverse primers. PCR amplification was performed by using a $\mathrm{T}_{100}{ }^{\mathrm{TM}}$ Thermal Cycler (Bio-Rad Laboratories, Inc., California, USA) with the following program: $94{ }^{\circ} \mathrm{C}$ for $2 \mathrm{~min}$, 35 cycles at $94^{\circ} \mathrm{C}$ for $30 \mathrm{~s}, 55^{\circ} \mathrm{C}$ for $30 \mathrm{~s}$, and $72{ }^{\circ} \mathrm{C}$ for $1 \mathrm{~min}$ with a final extension at $72{ }^{\circ} \mathrm{C}$ for $10 \mathrm{~min}$.

The PCR products were separated on $6 \%$ denaturing polyacrylamide gel and detected by silver nitrate staining in accordance with Sambrook and Russell (2001). The size of the DNA bands was estimated by using a 100 bp DNA ladder (Invitrogen, California, USA). The DNA patterns of the parents ( $R$ and $S$ ) and DNA bulks (RB and SB) were observed to identify polymorphic markers. The putative markers, together with six other previously identified markers that are linked to the major CLS resistance QTL qCLSC72V18 in the 'CN72' $\times$ 'V4718' cross, were then used to analyze individual RILs to evaluate linkage with CLS resistance. The six markers included 
three ISSR and ISSR-anchored resistance gene analog (ISSR-RGA) markers (I16274, I88656, and I35P716) and three SSR markers (CEDG008, CEDG084, and VR393 markers) (Tantasawat et al., 2020).

\section{Linkage and QTL analyses}

Clearly resolved DNA bands were compared between $\mathrm{R}$ and $\mathrm{RB}$ and between $S$ and SB and were scored manually by assigning scores of " 1 " and " 3 " to DNA fragments that were similar to the bands of the resistant parent and the susceptible parent, respectively. The association between the putative marker and CLS scores for 2016 and 2018 was determined through simple linear regression, recombination calculation, $x^{2}$ test for the segregation of markers, and logarithm of odds (LOD) analysis. The markers that were associated with CLS resistance were used to construct the genetic linkage map with QTL IciMapping 4.1 software (Meng et al., 2015). They were assigned to the linkage group (LG) with a minimum LOD of 3.0, and the markers were ordered by using the Anchor Order function. Genetic distance was calculated with the Kosambi mapping function (Kosambi, 1943). The location of the QTL that was associated with CLS resistance was determined by using QTL IciMapping 4.1 software with an inclusive composite interval mapping (ICIM) function ( $\mathrm{Li}$ et al., 2007). Permutation tests were run 10000 times at $P=0.01$ to determine the LOD score threshold for the QTL.

\section{Verification of markers associated with the CLS resistance gene}

The closest markers identified in this study were verified in 22 mungbean varieties/lines with known CLS reactions. These genotypes included certified Thai varieties ('CN36', 'CN72', 'CN84-1', and 'SUT1'), developed varieties/lines ('SUT4' and 'SUPER 5'), and introduced varieties/lines from the World Vegetable Center ('V4718', 'V4758', 'V4785', 'TAINAN SEL\#5', 'PUSA-105', 'VAR A-G',
'BARI MUNG 2', 'NM92', 'NM94', 'EG-MD6D', 'CES55', 'MG50-10A (Y)', 'BPI GLABROUS \#3', 'WALET', 'GELATIK', and 'KING'). The data on the CLS reactions of these mungbean varieties/lines were obtained from Chueakhunthod et al. (2020), who evaluated CLS resistance under field conditions in Thailand.

\section{RESULTS}

Three SSR primer pairs and one InDel primer pair were used in BSA to determine the possible CLS-resistance-linked markers. The Vr6gCLS037, Vr6gCLS133, and VrTAF5_indel markers generated polymorphic alleles between R and RB and between $S$ and $S B$, indicating their possible association with CLS resistance, whereas the Vr6gCLS085 marker was monomorphic. The two SSR markers and the InDel marker that were putatively linked to CLS resistance were preliminarily analyzed through simple linear regression to identify a correlation between the markers and CLS scores from 2016 and 2018 data. All of these three markers were found to be linked to CLS resistance $(P<0.01)$. Subsequently, they were further subjected to LOD, simple linear regression, and recombination analyses with both RIL populations $\left(F_{2: 9}\right.$ and $\left.F_{2: 10}\right)$ to verify their linkage with CLS resistance. Only the VrTAF5_indel marker had a $x^{2}$ with $1: 1$ ratio. The $R^{2}$ of the VrTAF5_indel marker was 0.481 for 2016 and 0.328 for 2018 with a LOD score of more than 3 for both years ( 9.89 and 5.11 for 2016 and 2018, respectively), suggesting that this marker is useful for CLS mapping.

The marker VrTAF5_indel and six previously identified markers were used to establish the genetic linkage map of the $F_{2: 9}$ and $F_{2: 10}$ RIL populations for CLS mapping. These seven markers were grouped into the same LG and spanned a chromosome length of $65.5 \mathrm{cM}$ (Figure 1). QTL analysis with the ICIM function on both RIL populations by using CLS scores for 2016 and 2018 revealed that the qCLSC72V18-1 locus for CLS resistance 


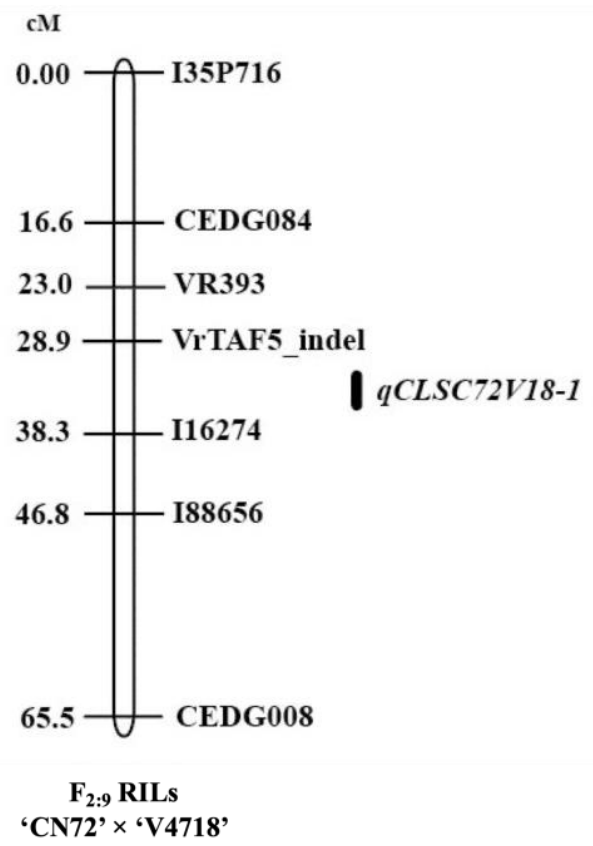

Figure 1. Illustration of the linkage map for the $F_{2: 9}$ RILs of the 'CN72' $\times$ 'V4718' cross.

Table 1. QTL detected for CLS resistance in $F_{2: 9}$ and $F_{2: 10}$ RIL populations derived from 'CN72' $\times$ 'V4718' cross. The QTL was detected by using the ICIM function.

\begin{tabular}{|c|c|c|c|c|c|c|c|}
\hline Population & Year & QTL name & Marker interval & $\begin{array}{l}\text { Position }^{a} \\
(\mathrm{cM})\end{array}$ & $\begin{array}{l}\text { LOD } \\
\text { score }^{b}\end{array}$ & $\begin{array}{l}\text { PVE }^{\mathrm{C}} \\
(\%)\end{array}$ & $\begin{array}{l}\text { Additive } \\
\text { effect }\end{array}$ \\
\hline$F_{2: 9}$ & 2016 & $q C L S C 72 V 18-1$ & $\begin{array}{l}\text { I16274- } \\
\text { VrTAF5_indel }\end{array}$ & 34.0 & 24.16 & 60.38 & -0.95 \\
\hline$F_{2: 10}$ & 2018 & qCLSC72V18-1 & $\begin{array}{l}\text { I16274- } \\
\text { VrTAF5 indel }\end{array}$ & 35.0 & 10.08 & 41.56 & -0.85 \\
\hline
\end{tabular}

${ }^{\text {aP }}$ osition on the linkage group.

${ }^{\mathrm{b}}$ Logarithm of odds (LOD) score explained by the QTL.

${ }^{\mathrm{c}}$ Percentage of phenotypic variance explained (PVE) by the QTL.

was flanked by the I16274 and VrTAF5_indel markers. This QTL, which was located at $34.0 \mathrm{cM}$, accounted for $60.38 \%$ of the variation in the disease responses observed in 2016 with the additive effect of -0.95 and accounted for a lower phenotypic variation of $41.56 \%$ in 2018; another QTL was detected at 35.0 CM with the additive effect of -0.85 (Table 1 ). These flanking markers will be applied to improve the CLS resistance of cultivated mungbean varieties in the future.
The I16274 and VrTAF5_indel markers were also tested on 22 mungbean varieties/lines with known CLS reactions (Table 2 ). The dominant I16274 marker amplified a 274 bp amplicon that was linked to CLS resistance (A_) in 'V4718', 'V4785', 'SUPER 5', 'TAINAN SEL\#5', 'VAR A-G', 'BARI MUNG 2', and 'WALET'. A 209 bp amplicon linked to CLS resistance was amplified by the codominant VrTAF5 indel marker in 'V4718' and 'SUPER 5' (BB) and in 'V4785' and 'GELATIK' (Bb). The remaining 14 
Table 2. Genotypic and phenotypic analyses of mungbean varieties/lines by using the I16274 and VrTAF5_indel markers.

\begin{tabular}{|c|c|c|c|}
\hline \multirow{2}{*}{ Varieties/Lines } & \multirow{2}{*}{ Phenotypes $^{a}$} & \multicolumn{2}{|c|}{ Genotypes $^{\mathrm{b}}$} \\
\hline & & I16274 & VrTAF5_indel \\
\hline V4718 & $\mathrm{R}$ & $A$ & BB \\
\hline V4785 & $\mathrm{R}$ & $A_{-}^{-}$ & $\mathrm{Bb}$ \\
\hline SUPER 5 & $\mathrm{R}$ & $A_{-}^{-}$ & $\mathrm{BB}$ \\
\hline V4758 & MR & $\mathrm{a} \bar{a}$ & $\mathrm{bb}$ \\
\hline TAINAN SEL\# 5 & MR & $A_{-}$ & bb \\
\hline CN36 & $\mathrm{S}$ & $\mathrm{a} \bar{a}$ & $\mathrm{bb}$ \\
\hline CN72 & $\mathrm{S}$ & aa & $\mathrm{bb}$ \\
\hline CN84-1 & $\mathrm{S}$ & aa & $\mathrm{bb}$ \\
\hline SUT1 & $\mathrm{S}$ & aa & $\mathrm{bb}$ \\
\hline SUT4 & $\mathrm{S}$ & aa & $\mathrm{bb}$ \\
\hline PUSA-105 & $\mathrm{S}$ & aa & $\mathrm{bb}$ \\
\hline VAR A-G & $\mathrm{S}$ & $A_{-}$ & $\mathrm{bb}$ \\
\hline BARI MUNG 2 & $\mathrm{~S}$ & $A_{-}^{-}$ & $\mathrm{bb}$ \\
\hline NM92 & $\mathrm{S}$ & $\mathrm{a} \bar{a}$ & $\mathrm{bb}$ \\
\hline NM94 & $\mathrm{S}$ & aa & $\mathrm{bb}$ \\
\hline EG-MD-6D & $\mathrm{S}$ & aa & $\mathrm{bb}$ \\
\hline CES55 & $\mathrm{S}$ & aa & $\mathrm{bb}$ \\
\hline MG50-10A (Y) & $\mathrm{S}$ & aa & $b b$ \\
\hline BPI GLABROUS \#3 & $\mathrm{S}$ & aa & $b b$ \\
\hline WALET & $\mathrm{S}$ & $A_{-}$ & $b b$ \\
\hline GELATIK & $\mathrm{S}$ & $\mathrm{a} \bar{a}$ & $\mathrm{Bb}$ \\
\hline KING & $\mathrm{S}$ & aa & $\mathrm{bb}$ \\
\hline
\end{tabular}

${ }^{\mathrm{a} C L S}$ responses were evaluated in 2018 and 2020 and classified into three classes: resistant $(R)=1-2.5$, moderately resistant $(M R)=2.6-3.4$, and susceptible $(S)=3.5-5.0$.

${ }^{\mathrm{b}}$ For the I16274 marker, A_: presence of 274 bp amplicon; aa: absence of 274 bp amplicon. For the VrTAF5_indel marker, BB: presence of 209 bp amplicon; bb: presence of 234 bp amplicon; Bb: presence of 209 and 234 bp amplicons.

genotypes, namely, 'V4758', 'CN36', 'CN72', 'CN84-1', 'SUT1', 'SUT4', 'PUSA105', 'NM92', 'NM94', 'EG-MD-6D', 'CES55', 'MG50-10A (Y)', 'BPI GLABROUS $\# 3$ ', and 'KING', lacked either of the amplicons (aabb). Some of the genotypes with aabb alleles from both markers, such as 'CN36', 'CN72', 'CN84-1', 'SUT1', and 'EG-MD-6D', have been reported to possess high-yielding potential under field conditions in Thailand (Chueakhunthod et al., 2020). The CLS resistance gene can be introduced into these genotypes through MAS by using the two tightly linked markers.

\section{DISCUSSION}

In breeding programs, the selection of desirable traits is typically hampered by the ambiguity of phenotypes and the requirement for specific environmental conditions. In MAS, molecular markers can be used to overcome these limitations. The mungbean accession 'V4718' has been found to provide stable resistance against CLS (Hartman et al., 1993; Chankaew et al., 2011, Tantasawat et al., 2020; Yundaeng et al., 2020). qCLS has been identified as the only locus that controls CLS resistance in 'V4718' 
(Chankaew et al., 2011; Tantasawat et al., 2020; Yundaeng et al., 2020). The segregation ratio in the $F_{2: 9}$ and $F_{2: 10}$ RIL populations derived from a cross between 'CN72' and 'V4718' is 1:1 for resistant and susceptible progenies (Tantasawat et al., 2020), indicating its qualitative nature with a dominantly inherited resistance. Therefore, the CLS resistance gene from 'V4718' can be transferred into a susceptible cultivated variety for the development of new CLS-resistant varieties via several conventional breeding methods, such as pedigree selection, bulk selection, single-seed descent, or backcrossing.

In mungbean, BSA has been generally applied to identify the markers linked to resistance against CLS, powdery mildew, and mungbean yellow mosaic virus, as well as bean bugs and bruchids (Selvi et al., 2006; Chen et al., 2007; Dhole and Reddy, 2013; Hong et al., 2015; Poolsawat et al., 2017; Sai et al., 2017; Dharajiya and Ravindrababu, 2019). In this study, BSA revealed that the marker VrTAF5_indel was significantly associated with the CLS resistance gene $(P<0.001)$.

qCLSC72V18-1, a major QTL that is located between markers VR393 and I16274, has been previously identified in the 'CN72' $\times$ 'V4718' cross (Tantasawat et al., 2020). Recently, Yundaeng et al. (2020) successfully identified the candidate gene VrTAF5 at the qCLS locus in the 'KPS1' $\times$ 'V4718' cross and developed gene-specific markers, including the VrTAF5_indel and Vr6gCLS133 markers that are tightly linked to $q C L S$. Therefore, the markers linked to VrTAF5 identified by Yundaeng et al. (2020) in the 'KPS1' $\times$ 'V4718' cross, as well as previously identified markers in this current cross, were simultaneously characterized to identify the close markers that are linked to the CLS resistance gene in our 'CN72' $\times$ 'V4718' cross. The ISSR marker I16274 and the InDel marker VrTAF5_indel were found to be tightly linked to $q C L S C 72 \mathrm{~V} 18-1$ with the calculated genetic distances of 4 and 5 cM, respectively, allowing only $0.40 \%$ recombination between both markers and the CLS resistance gene in MAS. The newly identified VrTAF5_indel marker appeared to be closer to qCLSC72V18-1 than VR393, which was identified in a previous study (Tantasawat et al., 2020) (Figure 1), and is thus more efficient for MAS than other markers. The genetic distance of the VrTAF5_indel marker from the CLS resistance gene in 'V4718' in our study (5 cM) differed from that in the work of Yundaeng et al. (2020), who found a distance of only $0.1 \mathrm{cM}$ likely because the maternal parents in the two populations were different as also observed by Arsakit et al. (2017). The I16274 and VrTAF5_indel markers would be very useful for accelerating the development of new mungbean cultivar(s) that are resistant to CLS disease. When we validated the I16274 and VrTAF5_indel markers in 22 mungbean varieties/lines with known CLS reactions, we found that 'V4718', 'V4785', and 'SUPER 5', the three mungbean lines with resistance response, all showed amplicons that were linked to CLS resistance. By contrast, 13 susceptible varieties/lines lacked the the resistance amplicons of $\mathrm{I} 16274$ and VrTAF5_indel markers, indicating that in contrast to 'V4718', they were polymorphic at these loci. Therefore, we can transfer the CLS resistance gene from the resistant line 'V4718' into these 13 varieties/lines through MAS. However, one resistance amplicon from either 116274 or VrTAF5_indel marker was found in the four remaining susceptible varieties/lines, namely, 'VAR A-G', 'BARI MUNG 2', 'WALET', and 'GELATIK', suggesting recombination in this region.

\section{CONCLUSIONS}

We successfully screened DNA polymorphism in the RILs of the 'CN72' $\times$ 'V4718' cross by using the SSR and InDel markers that were recently found to be closely linked to the CLS resistance gene in the 'KPS1' $\times$ 'V4718' cross. Our study identified a major QTL (qCLSC72V18-1) that accounted for $41.56 \%-60.38 \%$ of the 
variation in the CLS disease scores for 2016 and 2018. This QTL was flanked by the I16274 and VrTAF5_indel markers with the distances of 4 and $5 \mathrm{cM}$ from the CLS resistance gene, respectively. The markers that are closely linked to CLS resistance will be very useful for developing new CLS-resistant mungbean cultivar(s) through MAS.

\section{ACKNOWLEDGEMENTS}

This work was supported by the Agricultural Research Development Agency (Public Organization) and Suranaree University of Technology, Thailand. The authors also express their sincere appreciation to Peter C. Bint for editing the manuscript.

\section{REFERENCES}

Arsakit K, Papan P, Tharapreuksapong A, Tantasawat PA (2017). Simple sequence repeat markers associated with Cercospora leaf spot and powdery mildew resistance in mungbean (Vigna radiata L. Wilczek). Proceedings of the 2017 International Forum-Agriculture, Biology, and Life Science, Kyoto, Japan.

Asian Vegetable Research and Development Center - AVRDC (1980). AVRDC Progress Report 1980. Asian Vegetable Research and Development Center, Shanhua, Taiwan, Republic of China.

Asian Vegetable Research and Development Center - AVRDC (1984). AVRDC Progress Report 1984. Asian Vegetable Research and Development Center, Shanhua, Taiwan, Republic of China.

Chand R, Pal C, Singh V, Kumar M, Singh VK, Chowdappa P (2015). Draft genome sequence of Cercospora canescens: a leaf spot causing pathogen. Curr. Sci. 109(11): 2103-2110.

Chankaew S, Somta P, Sorajjapinun W, Srinives P (2011). Quantitative trait loci mapping of Cercospora leaf spot resistance in mungbean, Vigna radiata (L.) Wilczek. Mol. Breed. 28: 255-264.

Chen HM, Liu CA, Kuo CG, Chien CM, Sun HC, Huang CC, Lin YC, Ku HM (2007). Development of a molecular marker for a bruchid (Callosobruchus chinensis L.) resistance gene in mungbean. Euphytica 157: 113-122.

Chueakhunthod W, Jinagool W, Meecharoen K, Khwanman R, Pattanaram $\mathrm{P}$, Jantarat $N$, Palaphon $P$, Ngampongsai $S$, Tantasawat PA (2020). Genetic relationship of mungbean and blackgram genotypes based on agronomic and photosynthetic performance and SRAP markers. Not. Bot. Horti. Agrobo. 48(4): 1845-1861.

Dharajiya DT, Ravindrababu Y (2019). Identification of molecular marker associated with mungbean yellow mosaic virus resistance in mungbean [Vigna radiata (L.) Wilczek]. Vegetos 32(4): 532-539.

Dhole VJ, Reddy KS (2013). Development of a SCAR marker linked with a MYMV resistance gene in mungbean (Vigna radiata L. Wilczek). Plant Breed. 132: 127-132.

Hartman GL, Wang TC, Kim D (1993). Field evaluation of mungbeans for resistance to Cercospora leaf spot and powdery mildew. Int. J. Pest Manag. 39: 418421.

Hong MG, Kim KH, Ku JH, Jeong JK, Seo MJ, Park $\mathrm{CH}$, Kim YH, Kim HS, Kim YK, Baek SH, Kim DY, Park SK, Kim SL, Moon JK (2015). Inheritance and quantitative trait loci analysis of resistance gene to bruchid and bean bung in mungbean (Vigna radiata L. Wilczek). Plant Breed. Biotech. 3: 3946.

Iqbal SM, Zubair M, Haqqani AM (2004). Resistance in mungbean to Cercospora leaf spot disease. Int. J. Agric. Biol. 6: 792-793.

Khajudparn P (2009). Characters associated with yield potential and development of molecular markers for powdery mildew resistance in mungbean. Ph.D Thesis. School of Crop Production Technology, Suranaree University of Technology, Nakhon Ratchasima, Thailand.

Kosambi DD (1943). The estimation of map distances from recombination values. Ann. Euge. 12: 172-175.

Leabwon U, Oupadissakoon S (1984). Inheritance of resistance to Cercospora leaf spot in mungbean. Kasetsart $J$. (Nat. Sci.). 18: 14-19.

Li H, Ye G, Wang J (2007). A modified algorithm for the improvement of composite interval mapping. Genetics 175: 361-374. 
Meng L, Li H, Zhang L, Wang J (2015). QTL IciMapping: integrated software for genetic linkage map construction and quantitative trait locus mapping in biparental populations. Crop J. 3: 269283.

Mishra SP, Asthana AN, Yadav L (1988). Inheritance of Cercospora leaf spot resistance in mung bean, Vigna radiata (L.) Wilczek. Plant Breed. 100: 228229.

Nair RM, Pandey AK, War AR, Hanumantharao B, Shwe T, Alam AKMM, Pratap A, Malik $\mathrm{SR}$, Karimi R, Mbeyagala EK, Douglas CA, Rane J, Schafleitner R (2019). Biotic and abiotic constraints in mungbean production-progress in genetic improvement. Front. Plant Sci. 10: 1340-1363.

Poczai P, Varga I, Laos M, Cseh A, Bell N, Valkonen JPT, Hyvönen J (2013). Advances in plant gene-targeted and functional markers: a review. Plant Methods 9: 6-36.

Poolsawat O, Kativat C, Arsakit K, Tantasawat PA (2017). Identification of quantitative trait loci associated with powdery mildew resistance in mungbean using ISSR and ISSR-RGA markers. Mol. Breed. 37: 150-161.

Ruanpanun P, Somta P (2021). Identification and resistant characterization of legume sources against Meloidogyne incognita. J. Integr. Agric. 20(1): 168177.
Sai CB, Nagarajan $\mathrm{P}$, Raveendran $\mathrm{M}$, Rabindran R, Kannan Bapu JR, Senthil N (2017). Understanding the inheritance of mungbean yellow mosaic virus (MYMV) resistance in mungbean (Vigna radiata L. Wilczek). Mol. Breed. 37(5): 63-77.

Sambrook J, Russell DW (2001). Molecular cloning: a laboratory manual. ( $3^{\text {rd }}$ ed.). Cold Spring Harbor Laboratory Press, Cold Spring Harbor, New York.

Selvi R, Muthiah AR, Manivannan N, Raveendran TS, Manickam A, Samiyappan R (2006). Tagging of RAPD marker for MYMV resistance in mungbean [Vigna radiata (L.) Wilczek]. Asian J. Plant Sci. 5: 277-280.

Tantasawat PA, Poolsawat O, Arsakit K, Papan $P$ (2020). Identification of ISSR, ISSRRGA and SSR markers associated with Cercospora leaf spot resistance gene in mungbean. Int. J. Agric. Biol. 23(2): 447-453.

Wongpiyasatid A, Chotechuen S, Hormchan P, Srihuttagum M (1999). Evaluation of yield and resistance to powdery mildew, Cercospora leaf spot and cowpea weevil in mungbean mutant lines. Kasetsart J. (Nat. Sci.). 33(2): 204-215.

Yundaeng C, Somta P, Chen J, Yuan X, Chankaew S, Chen X (2020). Fine mapping of QTL conferring Cercospora leaf spot disease resistance in mungbean revealed TAF5 as candidate gene for the resistance. Theor. Appl. Genet. 134: 701-714. 